\title{
A novel approach in acidic disinfection through inhibition of acid resistance mechanisms; Maleic acid-mediated inhibition of glutamate decarboxylase activity enhances acid sensitivity of Listeria monocytogenes
}

Article

Accepted Version

Creative Commons: Attribution-Noncommercial-No Derivative Works 4.0

Paudyal, R., Barnes, R. H. and Karatzas, K. A. G. (2018) A novel approach in acidic disinfection through inhibition of acid resistance mechanisms; Maleic acid-mediated inhibition of glutamate decarboxylase activity enhances acid sensitivity of Listeria monocytogenes. Food Microbiology, 69. pp. 96-104. ISSN 0740-0020 doi: https://doi.org/10.1016/j.fm.2017.07.013 Available at https://centaur.reading.ac.uk/71438/

It is advisable to refer to the publisher's version if you intend to cite from the work. See Guidance on citing.

To link to this article DOI: http://dx.doi.org/10.1016/j.fm.2017.07.013

Publisher: Elsevier 
copyright holders. Terms and conditions for use of this material are defined in the End User Agreement.

\section{www.reading.ac.uk/centaur}

\section{CentAUR}

Central Archive at the University of Reading

Reading's research outputs online 
1 A novel approach in acidic disinfection through inhibition of acid resistance

2 mechanisms; Maleic acid-mediated inhibition of glutamate decarboxylase activity

3 enhances acid sensitivity of Listeria monocytogenes

4

5 Ranju Paudyal ${ }^{\text {a }}$ Ruth H. Barnes ${ }^{\text {a }}$ and Kimon Andreas G. Karatzas*a

6 a Department of Food and Nutritional Sciences, The University of Reading, PO Box 226,

$7 \quad$ Whiteknights, Reading RG6 6AP, UK.

8

9

10

* Corresponding author

11

14 Corresponding author address:

15 School of Chemistry, Food and Pharmacy

16 Department of Food \& Nutritional Sciences,

17 University of Reading,

18 Reading

19 RG6 6AD

20 UK

21

Fax. +44 1189310080

24

Email: k.karatzas@reading.ac.uk

25

26

Keywords: Listeria monocytogenes, maleic acid, glutamate decarboxylase, inhibition, GAD, biofilm 


\section{ABSTRACT}

Here it is demonstrated a novel approach in disinfection regimes where specific molecular acid resistance systems are inhibited aiming to eliminate microorganisms under acidic conditions. Despite the importance of the Glutamate Decarboxylase (GAD) system for survival of Listeria monocytogenes and other pathogens under acidic conditions its potential inhibition by specific compounds that could lead to its elimination from foods or food preparation premises has not been studied. The effects of maleic acid on the acid resistance of L. monocytogenes were investigated and found that it has a higher antimicrobial activity under acidic conditions than other organic acids, while this could not be explained by its pKa or $\mathrm{Ki}$ values. The effects were found to be more pronounced on strains with higher GAD activity. Maleic acid affected the extracellular GABA levels while it did not affect the intracellular. Maleic acid had a major impact mainly on GadD2 activity as also shown in cell lysates. Furthermore, it was demonstrated that maleic acid is able to partly remove biofilms of L. monocytogenes. Maleic acid is able to inhibit the GAD of L. monocytogenes significantly enhancing its sensitivity to acidic conditions and together with its ability to remove biofilms, make a good candidate for disinfection regimes. 


\section{INTRODUCTION}

Listeriosis caused by Listeria monocytogenes is the leading cause of death due to a food borne illness in the UK (Mook et al., 2011) and as such is a serious problem of Public Health affecting the Food Industry. L. monocytogenes is a facultative anaerobic bacterium that can be isolated from soil, water, animal feed, faeces and tissues from various invertebrates and vertebrate animals including humans (Cooper and Walker, 1998). This bacterium has the ability to proliferate in a wide range of temperatures even below zero (Hudson et al. 1994) while it can persist (Fagerlund et al., 2016; Holch et al., 2013) and it is difficult to control in food processing environments (Salyers and Whitt, 2002). Normally, sodium hypochlorite or benzalkonium chloride are used while to a lesser extend acidic disinfectants are also used (Barker and Park 2001; Zhang and Farber 1996). However, a major factor affecting the popularity of a specific class of disinfectants is their antimicrobial activity and here we present a concept that could significantly increase the antimicrobial activity of class of acidic disinfectants. This novel approach that could be used to eliminate L. monocytogenes involves specifically inhibiting acid resistance mechanisms in combination with acidic conditions. One such cellular target is the glutamate decarboxylase (GAD) system which is the most important acid resistance system in L. monocytogenes (Cotter et al., 2001a) that comprises three decarboxylases (GadD1, GadD2 and GadD3) and two antiporters, (GadT1 and GadT2; Cotter et al., 2005). The antiporters import extracellular glutamate, which is converted to GABA and $\mathrm{CO}_{2}$, with a subsequent removal of protons, $\mathrm{pH}$ increase and export of GABA in exchange of another glutamate molecule (Paudyal and Karatzas, 2016). Furthermore, the decarboxylases can remove protons through processing intracellular glutamate through the intracellular GAD system $\left(\mathrm{GAD}_{\mathrm{i}}\right.$; Karatzas et al., 2012). Therefore, affecting the activity of the GAD system could enhance the sensitivity to acid treatments, resulting in successful 
elimination from food processing environments and food contributing to the reduction of foodborne disease.

Previously, maleic acid has been shown to inhibit the GAD system of E. coli (Fonda, 1972) while we are not aware of any similar work on other bacteria. Lately, maleic acid has been proposed to substitute the more toxic EDTA in dentistry for plaque removal during implementation of root canals (Ballal et al., 2009b) while it has been shown to eradicate $E$. faecalis biofilms (Ferrer-Luque et al., 2010). Organic acids are commonly used in food preservation and in disinfection because of their antimicrobial effects and their low toxicity. An example is lactic acid and its salts that are widely used as antimicrobials in various food products, particularly in meat and poultry (Dibner and Buttin, 2002). However, the antimicrobial effectiveness of maleic acid and its mode of action have not been thoroughly investigated and this is what was attempted in the present study. Firstly, various organic acids such as succinic, acetic, lactic and maleic acid for their inhibitory effects against the growth of L. monocytogenes were investigated and maleic acid ranked last. Despite that, it ranked first in bactericidal activity against the same organism under acidic conditions. Subsequently, its mode of action was investigated through functional genomics and protein activity of the GAD system activity and its ability to remove biofilms of this organism as it has been shown to do in dental biofilms (Ballal et al., 2009a).

\section{MATERIALS AND METHODS}

\subsection{Bacterial strains and growth conditions.}

All strains (Table 1) were stored in cryovials with $7 \%$ DMSO at $-80^{\circ} \mathrm{C}$. Stock cultures from $80^{\circ} \mathrm{C}$ were passed onto Brain Heart Infusion (BHI) agar (LABM, Lancashire UK) and incubated at $37^{\circ} \mathrm{C}$ overnight. Three colonies from each plate were transferred with a loop in 3 
$\mathrm{ml}$ of sterile $\mathrm{BHI}$ (LAB M, Lancashire UK) and incubated overnight at $37^{\circ} \mathrm{C}$ with shaking (140 rpm). Subsequently, the overnight cultures were used to inoculate $20 \mathrm{ml}$ of sterile BHI medium (1\% inocula) in $250 \mathrm{ml}$ conical flasks and incubated overnight $(\sim 18 \mathrm{~h})$ at $37^{\circ} \mathrm{C}$ with shaking (140 rpm). These overnight cultures were used for all acid challenges and assays described below.

\subsection{Determination of Minimum Inhibitory Concentrations (MICs)}

Concentrations ranging from 0.5-6.9 mg/ml (4.31-60.30 mM) of maleic, succinic, lactic and acetic acid were prepared. BHI Broth prepared with different acids was inoculated with $1 \%$ inoculum of overnight cultures and $200 \mu \mathrm{l}$ of that were placed on 96-well plates. The growth was measured overnight in a Sunrise machine (Tecan, Mannedorf, Switzerland) operated by Magellan software (Tecan, Mannedorf, Switzerland) at $620_{\mathrm{nm}}$ with 20 min time intervals between measurements at $37^{\circ} \mathrm{C}$ to identify the MIC.

\subsection{Survival under acidic conditions}

Twenty $\mathrm{ml}$ cultures were prepared in BHI and grown in $250 \mathrm{ml}$ flasks overnight at $37^{\circ} \mathrm{C}$ with shaking. Acid challenge took place with the addition of $4.31 \mathrm{mM}$ of succinic, acetic, lactic, $\mathrm{HCl}$ and maleic acid or no acid with the subsequent adjustment of the $\mathrm{pH}$ to 3.3 with the addition of $1 \mathrm{M} \mathrm{HCl}$ for EGD-e WT and its gad mutants. For 10403S WT and gad mutants the concentration used was $8.6 \mathrm{mM}$ with adjustment of $\mathrm{pH}$ to 3 , as the above conditions used for EGD-e did not affect the survival of this strain which has previously been shown to be highly acid tolerant (Karatzas et al., 2012). Samples were obtained prior to pH adjustment and thereafter every $20 \mathrm{~min}$ up to $60 \mathrm{~min}$ and used to prepare 10 -fold serial dilutions which were plated onto $\mathrm{BHI}$ agar and incubated at $37^{\circ} \mathrm{C}$ overnight, and subsequently, colonies were 
counted to assess survival under lethal acidic conditions. All experiments were performed in triplicate.

\subsection{GABase assays}

GABase assay was used to determine the concentrations of intracellular GABA $\left(\mathrm{GABA}_{\mathrm{i}}\right)$ in 10403S and EGD-e and extracellular GABA $\left(\mathrm{GABA}_{\mathrm{e}}\right)$ in $10403 \mathrm{~S}$ and $\mathrm{LO} 28 . \mathrm{GABA}_{\mathrm{i}}$ was quantified as described by O'Byrne et al., (2011) while GABAe was quantified according to the method of Tsukatani et al., (2005) as modified by Karatzas et al., (2010). The GABAse reaction was monitored by the measurement of absorbance at 340nm every $2 \min$ for $3 \mathrm{~h}$ at $37^{\circ} \mathrm{C}$ using a Sunrise spectrophotometer (Tecan, Mannedorf, Switzerland) operated by were obtained from Sigma-Aldrich (Steinheim, Germany).

\subsection{GAD activity in protein lysates}

Cultures of 10403S and EGD-e were grown in BHI overnight and they were transferred in 50 $\mathrm{ml}$ centrifuge tubes supplemented with $10 \mu \mathrm{g} / \mathrm{ml}$ chloramphenicol to prevent any further protein translation and were centrifuged at $12,000 \mathrm{X} \mathrm{g}$ for $15 \mathrm{~min}$. Cell pellets were washed with sonication buffer as described previously (Abram et al., 2008; Boura et al., 2016) and final cell suspensions were incubated for $30 \mathrm{~min}$ with shaking at $37^{\circ} \mathrm{C}$. An Eppendorf tube was then filled with acid-washed glass beads (106 $\mu$ m diameter; Sigma-Aldrich, Steinheim, Germany) and $1 \mathrm{ml}$ of cell suspension was transferred to it. Samples were disrupted thrice by a Mini-Beadbeater (Biospec, Bartesville, USA) for $1 \mathrm{~min}$ and cooled for $1 \mathrm{~min}$ on ice. Then 0.1\% DNAse1 (Sigma-Aldrich, Saint Louis, USA) was added to the cell lysate, incubated at $37^{\circ} \mathrm{C}$ for $30 \mathrm{~min}$ with shaking and $1 \mathrm{ml}$ was transferred into Eppendorf tubes and centrifuged at 5,000 X $\mathrm{g}$ for $15 \mathrm{~min}$. The supernatant was then transferred to sterile Eppendorf tubes and 
$40 \mu \mathrm{l}$ of this was mixed with $450 \mu \mathrm{l}$ of pyridine hydrochloride buffer (P-HCl; Fonda, 1972) adjusted at $\mathrm{pH} 4.5$ and supplemented with $30 \mathrm{mM}$ glutamate with or without $8.6 \mathrm{mM}$ maleic acid. Subsequently, GABA levels were measured through GABase assays as described above. Previously, with the use of standard concentrations of GABA it was shown that maleic acid does not inhibit the activity of GABase.

\subsection{Determination of $G A B A$ by $G C-M S$}

As the activity of the GABase enzyme could be affected by various molecules present in the cultures or the supernatant or the bacterial lysates, GABA concentrations in randomly selected samples were also determined by gas chromatography - mass spectrometry as described previously by Elmore et al., (2005). Results were compared with those by GABase assay and in all cases levels were $\pm 5 \%$.

\subsection{Biofilm removal by maleic acid}

Biofilm formation and its removal by maleic acid was tested on 96-well flat bottom plates. Biofilm assay protocol was adapted from Borucki et al., (2003) as modified by Harvey et al., (2007) based on the original work of O'Toole and Kolter, (1998), with modifications. 10403S WT was inoculated in BHI at $1 \%$ and $200 \mu \mathrm{l}$ of that were placed in each well of the 96 well plates and incubated at $30^{\circ} \mathrm{C}$ static for 4 days. Subsequently, liquid cultures were discarded and biofilms were treated with different maleic acid concentrations $(0-17.23 \mathrm{mM})$ at $30^{\circ} \mathrm{C}$ for 2 min. Then, plates were washed twice with distilled water and were inverted on a paper towel to remove excess moisture followed by drying in a laminar flow hood. Biofilms were stained for 20 min with $0.1 \%$ aqueous solution of crystal violet and washed with distilled water three times followed by drying for $20 \mathrm{~min}$. The adhered biofilms were dissolved in 
$95 \%$ ethanol and the optical density was measured at $620 \mathrm{~nm}$ in a Tecan Sunrise microplate reader (Tecan UK Ltd, Theale, RG7 5AH UK) operated by Magellan software.

\subsection{Calculation of undissociated acids using Henderson-Hasselbalch equation}

The undissociated percentage of acid was determined according to the equation of Henderson-Hasselbalch as adapted by Wemmenhove et al., (2016).

$$
[\text { Undissociated acid }]=[\text { Total acid }] / 1+10^{(\mathrm{pH}-\mathrm{pKa})}
$$

Molarities of total acid of solutions were pre-set, $\mathrm{pH}$ values were those values determined prior and after incubation. pKa values were used as described in literature. pKa 1.9, 6.07 for maleic acid, 5.61,4.2 for succinic acid, 4.76 for acetic acid and 3.86, 15.1 for lactic acid.

\subsection{Statistical analysis}

In all cases, experiments were run at least in triplicate (unless stated), and the results were assessed with paired Student $t$ test. $P$ values lower than 0.05 indicated results that were statistically significant.

\section{RESULTS}

\subsection{MICs of different organic acids.}

The MICs of WT 10403S for maleic, succinic, lactic and acetic acid were $34 \mathrm{mM}, 25 \mathrm{mM}, 31$ $\mathrm{mM}$ and $30 \mathrm{mM}$ which corresponded to $\mathrm{pH}$ values prior to growth of 4.84, 5.14, 5.32 and 5.02 respectively (Table 2 ). Of all compounds tested, maleic acid was the least inhibitory despite acting at a lower $\mathrm{pH}(4.84)$ while succinic was the most inhibitory.

\subsection{Acid survival of WT $10403 S$ and EGD-e in the presence of different acids.}


To investigate the effect of different acids on acid survival, the most acid resistant (10403S) and the weakest (EGD-e) strain were challenged with $8.6 \mathrm{mM}$ and $4.3 \mathrm{mM}$ of each organic acid at pH 3 and 3.3 respectively. On both strains, maleic acid was the most bactericidal (Fig. 1A, B).

\subsection{Survival of WT 10403S, LO28 and EGD-e in the presence of maleic acid.}

Once it was determined that maleic acid was the most bactericidal, the survival of 10403S and two other strains of L. monocytogenes (LO28 WT and EGD-e) was assessed against 4.3 $\mathrm{mM}$ maleic acid at $\mathrm{pH}$ 3. In the absence of maleic acid, 10403S WT was the most resistant strain while LO28 WT intermediate and EGD-e WT the most sensitive (Fig. 2A, B, C). However, in the presence of maleic acid results were reversed, with the highest antimicrobial effect seen on 10403S WT followed by LO28 and EGD-e WT (Fig. 2A, B, C). As the GAD system is known to be the most important acid resistance mechanism in L. monocytogenes (Cotter et al., 2001b), the GABAe production of these strains was assessed and it was found that $10403 \mathrm{~S}$ had the most active $\mathrm{GAD}_{\mathrm{e}}$ followed by LO28 and by EGD-e, with the latter producing no $\mathrm{GABA}_{\mathrm{e}}$ (Fig. 2D).

\subsection{The role of GAD genes in the presence of maleic acid in EGD-e}

In the absence of maleic acid, there was no significant difference between the strains at $\mathrm{pH}$ 3.3 after $60 \mathrm{~min}$ (Fig. 3A, B) while when $4.3 \mathrm{mM}$ maleic acid was added, EGD-e WT, $\triangle g a d D 1, \Delta g a d D 2$ and $\triangle g a d D 3$ showed 2.46, 2.79, 3.8 and 5.2 log reduction respectively (Fig. 3A). Thus, $\Delta g a d D 3$ was the most sensitive strain suggesting that GadD3 is the most important GAD system component for survival either in the presence of maleic acid (Fig. 3A) or in its absence (Fig. 3B). This coincides with the lower level of GABA $A_{i}$ in the $\triangle$ gadD3 while there was no statistically significant difference in the levels of $\mathrm{GABA}_{\mathrm{i}}$ between the 
other mutants and the WT (Fig. 3C). Interestingly, although the presence of maleic acid enhanced the acid sensitivity of all strains (Fig. 3A), it did not seem to affect the GABA levels (Fig. 3C).

\subsection{The role of GAD genes in the presence of maleic acid in $10403 S$}

In presence of $8.6 \mathrm{mM}$ maleic acid at $\mathrm{pH} 3$, WT, $\Delta \operatorname{gadD1}, \Delta \operatorname{gadD} 2, \Delta \operatorname{gadD} 3$ showed 2.0, 2.55, 2.61 and $3.28 \log$ reduction respectively (Fig. 4A). In the presence of maleic acid, $\triangle g a d D 2$ showed a rapid inactivation resulting in approximately $3.2 \log$ reduction of the $\mathrm{CFU} / \mathrm{ml}$ the first $20 \mathrm{~min}$, followed by a minor reduction of $0.5 \mathrm{log} \mathrm{CFU} / \mathrm{ml}$ the next $40 \mathrm{~min}$. $\Delta g a d D 2$ was the most sensitive strain followed by $\Delta$ gadD3 (Fig. 4A) and the relative sensitivity between strains was similar in the absence of maleic acid (Fig. 4B).

To understand the role of the components of the GAD system genes in the presence of maleic acid, the GAD activity of the 10403S WT and its isogenic GAD mutants was assessed. $\triangle$ gadD2 was found to produce no GABA $($ Fig. 4C) and very low levels of GABA $($ Fig. 4D) both in the presence or absence of maleic acid. All other gene deletions did not seem to have any major effect on $\mathrm{GABA}_{i}$ levels, while similarly to EGD-e, maleic acid did not seem to

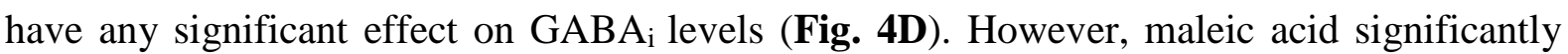
reduced $\mathrm{GABA}_{\mathrm{e}}$ levels by $61 \%(\mathrm{P}<0.05$; paired $\mathrm{T}$-test), $30 \%$ and $38 \%$ in the $\mathrm{WT}, \Delta$ gadD1 and $\triangle g a d D 3$ respectively, while $\triangle g a d D 2$ was unable to produce any GABA $($ Fig. 4C).

\subsection{GAD activity in protein lysates.}

GAD activity was determined in protein lysates through the assessment of GABA production in the presence of glutamate. Our results show that in 10403SWT, maleic acid resulted in a statistically significant decrease of GAD activity by $21 \%(\mathrm{P}=0.0053$; Fig. 5). It was also attempted unsuccessfully to setup a similar assay for EGD-e but despite trying different $\mathrm{pH}$ 
values we were unable to detect any GABA (data not shown). This might be due various reasons, such as low levels of GadD3 that cannot result in detectable GABA, inability to recover GadD3 or specific unknown conditions that are required for GadD3 function.

\subsection{Biofilm Formation.}

In all cases the $\mathrm{OD}_{(620 \mathrm{~nm})}$ measuring the concentration of crystal violet corresponding to the concentration of cells in the biofilm present on polystyrene was slightly lower with statistical significance in the presence of maleic acid than the control (Fig. 6). This effect was seen even at low maleic acid concentrations $(<17.23 \mathrm{mM})$ suggesting that the latter could partly remove biofilms of L. monocytogenes.

\subsection{Calculation of the percentage of undissociated acid}

One of the two pKas of maleic acid is extremely low (1.9) compared to other weak acids resulting in a low percentage of undissociated $(8.01-0.04 \%, \mathrm{pH} 3-5.32)$ and a significant percentage of dissociated molecules $(91.99-99.96 \%$, pH $3-5.32$; Table 3). As seen in (Table 3), in contrast to the other acids, the majority of the molecules are in dissociated form in all $\mathrm{pH}$ values used.

\section{DISCUSSION}

In the present study, a novel and more targeted approach for the disinfection of $L$. monocytogenes that might apply to other pathogens is investigated. The concept relies on the inhibition of major acid resistance systems such as the glutamate decarboxylase system in combination with the application of acidic conditions. This approach could be followed in the inactivation of various other pathogenic organisms possessing the GAD system such as $E$. coli (Waterman and Small, 2003), Shigella flexneri (Waterman and Small, 2003), 
278 (Feehily et al., 2013), B. abortus (Roop et al., 2003) or even spoilage organisms such as several species of lactic acid bacteria (Su et al., 2011). Further research could identify more inhibitors of the GAD system or other amino acid decarboxylase systems.

Initially the growth of $10403 \mathrm{~S}$ WT in the presence of different organic acids namely succinic, lactic, maleic and acetic was investigated. The MIC of maleic acid was $34 \mathrm{mM}$ which was the highest compared to the MIC of the other acids suggesting that it was the least inhibitory

(Table 3). This should be expected as one of the pKas of maleic acid is as low as 1.9 and at relatively mild acidic conditions, such as those used in the MIC experiments ( $\mathrm{pH} \sim 5$ ), only a small percentage of the acid would be in the undissociated form $(<0.08 \%$; Table 3$)$ which is more antimicrobial. The other carboxyl would have a minor influence in the formation of undissociated molecules as the first carboxyl dissociates almost fully (>92\%) in pH values above 3 . In contrast, the other acids have a significantly higher percentage of undissociated molecules than maleic acid (Table 3).

Subsequently, the bactericidal activity of these acids focusing on the survival of two $L$. monocytogenes strains EGD-e WT (acid sensitive) and 10403S WT (acid resistant) was studied at pH 3.3 and 3 respectively. (Fig. 1A, B). Interestingly, maleic acid possessed a bactericidal form compared to that of maleic acid. Despite that, maleic acid excreted significantly stronger bactericidal activity than the other acids at the same $\mathrm{pH}$. This suggests that maleic acid could be used to enhance the antibacterial effects of acidic environments. 
301 Furthermore, the bactericidal effects of maleic acid were not due to the classical mechanism of release of protons intracellularly by undissociated molecules of maleate entering the cell. In further experiments, the bactericidal effect of maleic acid at $\mathrm{pH} 3$ in three different strains of L. monocytogenes was investigated. We used L. monocytogenes $10403 \mathrm{~S}$ which is one of the most acid resistant strains described in literature, LO28 which has a moderate acid resistance and EGD-e which is one of the most acid sensitive strains described (Feehily et al., 2014; Fig. 2). Interestingly, the bactericidal activity of maleic acid was more pronounced with the most acid resistant strain of L. monocytogenes (10403S; Fig. 2C) less pronounced with moderately acid resistant strain (LO28; Fig. 2B) and least pronounced with the least acid resistant strain (EGD-e; Fig. 2A). It could be said, that bactericidal effects seemed to be more pronounced the more acid resistant a strain was, but also the higher its GAD activity was (Fig. 2D) as these two latter characteristics corresponded well for these 3 strains (Fig. 2). This suggested that maleic acid could be involved with the function of the GAD system in $L$. monocytogenes.

To investigate this hypothesis, it was attempted to see the effect of maleic acid on strains carrying deletions of the GAD genes. The EGD-e strain does not use the GADe system mediated mainly by GadD2 but relies on $\mathrm{GAD}_{\mathrm{i}}$ mediated mainly by GadD3 which utilises intracellular pools of glutamate (Feehily et al., 2014; Karatzas et al., 2012). This is reflected in the results as the $\triangle$ gadD3 was more sensitive than all other strains in the presence or absence of maleic acid (Fig. 3A, B). This is explained by the inability of this mutant to utilise the $\mathrm{GAD}_{\mathrm{i}}$ system which mainly comprises GadD3 as seen by the complete absence of intracellular GABA either in the presence or absence of maleic acid (Fig. 3C). In agreement with previous work, (Feehily et al., 2014) GadD1 seems to be completely inactive as the corresponding mutant showed similar survival to the wild type (Fig. 3A). It should be mentioned here that GadD1 of L. monocytogenes is a designated glutamate decarboxylase 
only on the basis of genetic similarity and no worker neither we have ever shown it to possesses such an activity. Therefore, it is still debatable if GadD1 is a glutamate decarboxylase. The survival of the other mutants (Fig. 3A) and the levels of GABA did not show any possible inhibition by maleic acid. However, it should be stated that GABA levels are also affected by its catabolism through the GABA shunt and therefore they do not reflect GAD activity. Similarly, there was no effect of maleic acid or mutations (apart from gadD2) on the levels of $\mathrm{GAD}_{\mathrm{i}}$ in $10403 \mathrm{~S}$.

On the other hand, there was a major effect of maleic acid on $\mathrm{GAD}_{\mathrm{i}}$ activity in $10403 \mathrm{~S}$ as all mutants apart from $\triangle g a d D 2$, which does not possess $\mathrm{GAD}_{\mathrm{e}}$ activity, showed a reduced $\mathrm{GAD}_{\mathrm{e}}$ activity in the presence of maleic acid (Fig. 4C). This reduced $G^{2} D_{\text {e }}$ activity resulted in increased inactivation in the presence of maleic acid (Fig. 4A). Increased inactivation was observed also in the case of EGD-e, but this was more enhanced with 10403S. This was clearly due to the inhibition of GadD2 in 10403S, while inhibition of maleic acid towards GadD3 might not be so strong.

To investigate further the effect of maleic acid on the GAD system, its effect on the GAD activity in lysates of $10403 \mathrm{~S}$ in the presence or absence of maleic acid was investigated. In these experiments, it was clearly demonstrated that maleic acid inhibits GAD activity by $25 \%$ $(\mathrm{P}=0.0053$; Fig. 5). Furthermore, our experiments showed that maleic acid acts specifically on the glutamate decarboxylase and not on the antiporter which is a membrane protein and thus was removed in the process of producing the lysate. It has been shown in E. coli, that the glutamate decarboxylase enzymes (GadD1 and GadD2) are in the cytosol at neutral $\mathrm{pH}$ and undergoes conformational change under low $\mathrm{pH}$ triggering their move towards the membrane (Capitani et al., 2003). Previously, it has been shown that maleic acid inhibits glutamate decarboxylase activity in E. coli (Fonda, 1972) and in Curcurbita moschata (Ohno Mei, 
1962) however, to our knowledge this is the first time it is shown to inhibit the GAD activity in any other bacterium than $E$. coli.

The ability of various acids to inhibit the GAD system of $E$. coli has been studied previously (Fonda, 1972) and estimated the Ki of various acids. The Ki is the measure of the inhibition of the GAD enzyme and the $\mathrm{Ki}$ of succinate is lower than that of maleic for the GAD of $E$. coli. However, in our work maleic showed a much stronger effect on the cells of $L$. monocytogenes. However, the final effect is not only the result of the actual inhibition conferred, but also the ability of the compound to enter the cell.

Furthermore, since GadD2 is the main determinant of GAD system in $10403 \mathrm{~S}$ it could be said that maleate that enters the cell inhibits specifically GadD2 activity. Unfortunately, after several attempts it was impossible to identify the optimum $\mathrm{pH}$ for the $\mathrm{GAD}_{\mathrm{i}}$ system of EGD-e comprising mainly GadD3, to see if there is any inhibitory effect of maleic acid in this strain as well. We speculated that the optimum $\mathrm{pH}$ of $\mathrm{GAD}_{\mathrm{i}}$ driven mainly by GadD3 in EGD-e is higher than that of GadD2 in 10403S ( $\mathrm{pH} 4.5$ ). However, increasing the $\mathrm{pH}$ did not result in any measurable Gad activity.

Finally, the possibility of using maleic acid to remove biofilms of L. monocytogenes was investigated. Previously, it has been shown that maleic acid is able to remove E. faecalis biofilms and it has been suggested to replace EDTA as an irrigant in dentistry due to its lower toxicity (Ferrer-Luque et al., 2010). Furthermore, L. monocytogenes is known to form biofilms which are difficult to remove and contribute significantly in the appearance of various outbreaks and individual cases of this pathogen. Our results show that maleic acid is able to partly remove biofilms of $L$. monocytogenes at very low levels $(1-2 \mathrm{mM})$ and in combination with its ability to enhance the sensitivity of this pathogen in acidic environments it could be used in disinfections regimes for this purpose. 


\section{CONCLUSIONS}

376 Overall our work showcases a novel approach in disinfection regimes through inhibition of specific molecular acid resistance systems in combination with acidic conditions. Normally the inhibitor of the acid resistance system and in this specific case, the maleic acid which inhibits the GAD system, could be added in minor concentrations and bring about major reduction in the bacterial numbers. If this property is combined with biofilm removal, it could contribute in the removal of pathogens such L. monocytogenes and other microorganisms from food and food premises.

\section{ACKNOWLEDGEMENTS}

The authors would like to thank all colleagues at the Microbiology research team, Food and Nutritional Sciences, University of Reading. The help of Marcia Boura and our deceased colleague Dr. Bernard Mackey in revising parts of the manuscript has been of great value. The work was supported by a Marie Curie European Reintegration Grant (ERG 265154), awarded to Dr. K.A.G. Karatzas and funds from the University of Reading.

\section{REFERENCES}

Abram, F., Wan-Lin, S., Wiedmann, M., Boor, K.J., Coote, P., Botting, C., Karatzas, K. A G., O’Byrne, C.P., 2008. Proteomic analyses of a Listeria monocytogenes mutant 
efficacy of maleic acid and ethylenediaminetetraacetic acid in smear layer removal from instrumented human root canal: a scanning electron microscopic study. J. Endod. 35, $1573-6$.

Ballal, N.V., Kundabala, M., Bhat, S., Rao, N., Rao, B.S.S., 2009b. A comparative in vitro evaluation of cytotoxic effects of EDTA and maleic acid: root canal irrigants. Oral Surg. Oral Med. Oral Pathol. Oral Radiol. Endod. 108, 633-8.

Borucki, M.K., Peppin, J.D., White, D., Call, D.R., Loge, F., 2003. Variation in Biofilm Formation among Strains of Listeria monocytogenes. Appl. Environ. Microbiol. 69.

Boura, M., Keating, C., Royet, K., Paudyal, R., O’Donoghue, B., O’Byrne, C.P., Karatzas, K.A.G., 2016. The presence of SigB in Listeria monocytogenes strains EGD-e and 10403S leads to hypersensitivity to hydrogen peroxide in stationary phase under aerobic conditions. Appl. Environ. Microbiol. 353, AEM.00709-16.

Capitani, G., Biase, D. De, Aurizi, C., Gut, H., Bossa, F., Gru, M.G., Ch-, È., 2003. Crystal structure and functional analysis of Escherichia coli glutamate decarboxylase 22, 40274037.

Cole, S.T., Brosch, R., Parkhill, J., Garnier, T., Churcher, C., Harris, D., Gordon, S. V, Eiglmeier, K., Gas, S., Barry, C.E., Tekaia, F., Badcock, K., Basham, D., Brown, D., Chillingworth, T., Connor, R., Davies, R., Devlin, K., Feltwell, T., Gentles, S., Hamlin, N., Holroyd, S., Hornsby, T., Jagels, K., Krogh, a, McLean, J., Moule, S., Murphy, L., Oliver, K., Osborne, J., Quail, M. a, Rajandream, M. a, Rogers, J., Rutter, S., Seeger, K., Skelton, J., Squares, R., Squares, S., Sulston, J.E., Taylor, K., Whitehead, S., Barrell, B.G., 1998. Deciphering the biology of Mycobacterium tuberculosis from the complete genome sequence. Nature 393, 537-544.

Cooper, J., Walker, R.D., 1998. Listeriosis. Vet. Clin. North Am. Food Anim. Pract. 14, 113125. 
425 Cotter, P.D., Gahan, C.G., Hill, C., 2001a. A glutamate decarboxylase system protects Listeria monocytogenes in gastric fluid. Mol. Microbiol. 40, 465-475.

427

Cotter, P.D., O'Reilly, K., Hill, C., 2001b. Role of the glutamate decarboxylase acid resistance system in the survival of Listeria monocytogenes $\mathrm{LO} 28$ in low $\mathrm{pH}$ foods. J. Food Prot. 64, 1362-1368.

Cotter, P.D., Ryan, S., Gahan, C.G.M., Hill, C., 2005. Presence of GadD1 Glutamate Decarboxylase in Selected Listeria monocytogenes Strains Is Associated with an Ability To Grow at Low pH. Appl. Environ. Microbiol. 2005, 71(6):2832.

Dibner, J.J., Buttin, P., 2002. Use of Organic Acids as a Model to Study the Impact of Gut Microflora on Nutrition and Metabolism 1. J. Appl. Poult. Res. 11:453-463.

Elmore, J.S., Koutsidis, G., Dodson, A.T., Mottram, D.S., Wedzicha, B.L., 2005. Measurement of acrylamide and its precursors in potato, wheat, and rye model systems. J. Agric. Food Chem. 53, 1286-1293.

Fagerlund, A., Langsrud, S., Schirmer, B.C.T., Møretrø, T., Heir, E., 2016. Genome analysis of Listeria monocytogenes sequence type 8 strains persisting in salmon and poultry processing environments and comparison with related strains. PLoS One 11, 1-22.

Feehily, C., Finnerty, A., Casey, P.G., Hill, C., Gahan, C.G.M., O’Byrne, C.P., Karatzas, K.A.G., 2014. Divergent Evolution of the Activity and Regulation of the Glutamate Decarboxylase Systems in Listeria monocytogenes EGD-e and 10403S: Roles in Virulence and Acid Tolerance. PLoS One 9, e112649.

Feehily, C., O’Byrne, C.P., Karatzas, K.A.G., 2013. Functional $\gamma$-Aminobutyrate Shunt in Listeria monocytogenes: role in acid tolerance and succinate biosynthesis. Appl. Environ. Microbiol. 79, 74-80.

Ferrer-Luque, C.M., Arias-Moliz, M.T., González-Rodríguez, M.P., Baca, P., 2010. Antimicrobial activity of maleic acid and combinations of cetrimide with chelating 

agents against Enterococcus faecalis biofilm. J. Endod. 36, 1673-5.

451

Fonda, M.L., 1972. Glutamate decarboxylase. Substrate specificity and inhibition by carboxylic acids. Biochemistry 11, 1304-9.

Harvey, J., Keenan, K.P., Gilmour, A., 2007. Assessing biofilm formation by Listeria monocytogenes strains. Food Microbiol. 24, 380-392.

Holch, A., Webb, K., Lukjancenko, O., Ussery, D., Rosenthal, B.M., Gram, L., 2013. Genome sequencing identifies two nearly unchanged strains of persistent Listeria monocytogenes isolated at two different fish processing plants sampled 6 years apart. Appl. Environ. Microbiol. 79, 2944-2951.

Hudson, J.A., Mott, S.J., Penney, N., 1994. Growth of Listeria monocytogenes, Aeromonas hydrophila and Yersinia enterocolitica on vacuum and saturated carbon dioxide controlled atmosphere-packaged sliced roast beef. J. Food Prot. 57(3), 204-208.

Karatzas, K.A.G., Brennan, O., Heavin, S., Morrissey, J., O’Byrne, C.P., 2010. Intracellular accumulation of high levels of gamma-aminobutyrate by Listeria monocytogenes $10403 \mathrm{~S}$ in response to low $\mathrm{pH}$ : uncoupling of gamma-aminobutyrate synthesis from efflux in a chemically defined medium. Appl. Environ. Microbiol. 76, 3529-37.

Karatzas, K.A.G., Suur, L., O’Byrne, C.P., 2012. Characterization of the intracellular glutamate decarboxylase system: analysis of its function, transcription, and role in the Acid Resistance of Various Strains of Listeria monocytogenes. Appl. Environ. Microbiol. 78, 3571-9.

Mook, P., O’Brien, S.J., Gillespie, I.A., 2011. Concurrent conditions and human listeriosis, England, 1999-2009. Emerg. Infect. Dis. 17, 38-43.

O’Byrne, C.P., Feehily, C., Ham, R., Karatzas, K. a G., 2011. A modified rapid enzymatic microtiter plate assay for the quantification of intracellular $\gamma$-aminobutyric acid and succinate semialdehyde in bacterial cells. J. Microbiol. Methods 84, 137-9. 
475 O’Toole, G.A., Kolter, R., 1998. Initiation of biofilm formation in Pseudomonas fluorescens

476

477

478

479

480

481

482

483

484

485

486

487

488

489

490

491

492

493

494

495

496

497

498

499

WCS365 proceeds via multiple, convergent signalling pathways: A genetic analysis. Mol. Microbiol. 28, 449-461.

Ohno Mei, K.O., 1962. Studies on the Inhibition of Glutamic Acid Decarboxylase of Squash 51.

Paudyal, R., Karatzas, K.A.G., 2016. Chapter 10 - Stress adaptation of Listeria monocytogenes in acidic ready-to-eat products A2 - Kotzekidou, Parthena BT - Food Hygiene and Toxicology in Ready to Eat Foods. Academic Press, San Diego, pp. 167182.

Roop, R.M. 2nd, Gee, J.M., Robertson, G.T., Richardson, J.M., Ng, W.-L., Winkler, M.E., 2003. Brucella stationary-phase gene expression and virulence. Annu. Rev. Microbiol. $57,57-76$.

Salyers, A.A., Whitt, D.D., 2002. Bacterial Pathogenesis: A Molecular Approach, Bacterial Pathogenesis: A Molecular Approach. ASM Press.

Su, M.S., Schlicht, S., Gänzle, M.G., 2011. Contribution of glutamate decarboxylase in Lactobacillus reuteri to acid resistance and persistence in sourdough fermentation. Microb. Cell Fact. 10, S8.

Tsukatani, T., Higuchi, T., Matsumoto, K., 2005. Enzyme-based microtiter plate assay for $\gamma$ aminobutyric acid: Application to the screening of $\gamma$-aminobutyric acid-producing lactic acid bacteria. Anal. Chim. Acta 540, 293-297.

Waterman, S.R., Small, P.L.C., 2003. The glutamate-dependent acid resistance system of Escherichia coli and Shigella flexneri is inhibited in vitro by L-trans-pyrrolidine-2,4dicarboxylic acid. FEMS Microbiol. Lett. 224, 119-125.

Wemmenhove, E., van Valenberg, H.J.F., Zwietering, M.H., van Hooijdonk, T.C.M., WellsBennik, M.H.J., 2016. Minimal inhibitory concentrations of undissociated lactic, acetic, 
500

501

502

503

504

505

506

507

508

509

510

511

512

513

514

515

516

517

518

519

520

521

522

citric and propionic acid for Listeria monocytogenes under conditions relevant to cheese. Food Microbiol. 58, 63-67.

Zhang, S. and Farber J.M. 1996. The effects of various disinfectants against Listeria monocytogenes on fresh-cut vegetables. Food Microbiol 13(4), 311-321.

\section{LEGENDS TO THE FIGURES}

Fig. 1 (A) Survival of EGD-e WT in presence of concentration of $0.5 \mathrm{mg} / \mathrm{ml} \mathrm{maleic}$, succinate, $\mathrm{HCl}$, acetate and lactate grown in BHI. Subsequently, cultures were acid challenged at pH 3.3 with the addition of 1M HCl. (B) Survival of 10403S WT in presence of $1 \mathrm{mg} / \mathrm{ml}$ concentration of maleic, succinate, $\mathrm{HCl}$, acetate and lactate grown in $\mathrm{BHI}$. Subsequently, cultures were acid challenged at $\mathrm{pH} 3$ with the addition of $1 \mathrm{M} \mathrm{HCl}$. Both (A) and (B) cells were grown overnight until stationary phase $(\sim 18 \mathrm{~h})$ at $37^{\circ} \mathrm{C}$ with shaking. D.L denotes detection limit of the experimental setup.

Fig. 2 Survival of WT EGD-e (A), LO28 (B), 10403S (C) respectively. Strains were treated without and with $4.3 \mathrm{mM}$ of maleic acid grown in BHI until stationary phase $(\sim 18 \mathrm{~h})$ at $37^{\circ} \mathrm{C}$ with shaking. Subsequently, cultures were acid challenged at $\mathrm{pH} 3$ with the addition of $1 \mathrm{M}$ HCl. (D) Extracellular $\mathrm{GABA}_{\mathrm{e}}$ through time (min) following the adjustment of $\mathrm{pH} 4.2$ of overnight cultures of WT EGD-e, LO28 and 10403S grown until stationary phase in BHI.

Fig. 3 Survival of stationary phase cultures of EGD-e WT and its gad mutants after exposure at $\mathrm{pH} 3.3$ for $60 \mathrm{~min}$ in the presence or absence of $4.3 \mathrm{mM}$ of maleic acid (A), or in the absence of maleic acid at pH 2.5 (B). (C) GABA $_{i}$ of overnight stationary phase cultures of WT EGD-e and its isogenic gad mutants following exposure at $\mathrm{pH} 4.2$ for $60 \mathrm{~min}$, in the presence or absence of $4.3 \mathrm{mM}$ maleic acid. Cultures were grown in $\mathrm{BHI}$ for $\sim 18 \mathrm{~h}$ at $37^{\circ} \mathrm{C}$ 
525

526

527

528

529

530

531

532

533

534

535

536

537

538

539

540

541

542

543

544

with shaking, $\mathrm{pH}$ was adjusted with the addition of $1 \mathrm{M} \mathrm{HCl}$, while asterisks represent a statistically significant difference $(\mathrm{P}<0.05)$.

Fig. 4 Survival of stationary phase cultures of 10403S WT and its isogenic gad mutants at pH 3 in the presence or absence of $8.6 \mathrm{mM}$ of maleic acid after $40 \mathrm{~min}(\mathrm{~A})$ or, in the absence of maleic acid at $\mathrm{pH} 2.5$ for 60 min (B). Concentration of $\mathrm{GABA}_{e}(\mathrm{C})$ and $\mathrm{GABA}_{\mathrm{i}}(\mathrm{D})$ in stationary phase cultures of $10403 \mathrm{~S}$ WT and its gad mutants after $60 \mathrm{~min}$ at $\mathrm{pH} 4.2$ in the presence or absence of $8.6 \mathrm{mM}$ maleic acid. Intracellular $\mathrm{GABA}_{\mathrm{i}}$. Cultures were grown in BHI for $\sim 18 \mathrm{~h}$ at $37^{\circ} \mathrm{C}$ with shaking, $\mathrm{pH}$ was adjusted with the addition of $1 \mathrm{M} \mathrm{HCl}$, while asterisks represent a statistically significant difference $(\mathrm{P}<0.05)$.

Fig. $510403 \mathrm{~S}$ WT cells grown overnight until stationary phase $(\sim 18 \mathrm{~h})$ at $37^{\circ} \mathrm{C}$ with shaking. Lysates were produced and GAD activity was monitored in them through GABA measurements. Asterisk represents statistically significant difference $(\mathrm{P}<0.05)$.

Fig. 6 Average optical densities of crystal violet-stained biofilms of L. monocytogenes 10403S WT after growth in 96 well plates at $30^{\circ} \mathrm{C}$ without shaking. Error bars represent standard deviation from triplicate observations and asterisks represents statistically significant difference $(\mathrm{P}<0.05)$ with the control containing no maleic acid. 